\section{Uso de marcadores químicos no estudo da diversidade genética de Ocimum gratissimum L. ${ }^{1}$}

\author{
Roberto F. Vieira ${ }^{\text {**; }}$ Renée J. Grayer ${ }^{2}$; Alan Patonb ${ }^{2}$; James E. \\ Simon $^{3}$
}

1 Embrapa Recursos Genéticos e Biotecnologia, CP 02372, 70770-990, Brasilia, DF, Brazil

${ }^{2}$ Royal Botanical Gardens, Kew, Richmond, Surrey, TW9 3AB, U.K.

${ }^{3}$ Cook College, Rutgers University, New Brunswick, NJ, 089018520, USA.

\section{Introdução}

As alfavacas, ou alfavacão (Ocimum gratissimum L., Lamiaceae), é uma espécie comumente utilizada na medicina popular na América do $\mathrm{Sul}^{2}$, e Africa ${ }^{12,13}$. Esta espécie é também uma importante fonte comercial de eugenol na Índia, onde vem sendo cultivada ${ }^{19}$.

O centro de origem de Ocimum gratissimum é a África, e apesar de muitos estudos a respeito dos constituintes químicos de seus óleos essenciais, a taxonomia infraespecífica desta espécie permanece ainda confusa ${ }^{15}$. A espécie é subdividida em duas variedades: $O$. gratissimum var. gratissimum, a qual possui folhas e ramos pubescentes, e inflorescência densa; e $O$. gratissimum var. macrophyllum, que apresenta folhas e ramos glabros, com inflorescência aberta ${ }^{1,15}$. O. gratissimum apresenta uma larga variabilidade morfologica, especialmente em relação ao indumento das folhas e inflorescências, o que torna difícil o uso destes caracteres como indicador taxonômico.

Guenther ${ }^{10}$ relata que os óleos essenciais de $O$. gratissimum podem ser divididos em dois grupos, o primeiro com alto teor de eugenol, e o segundo com alto teor de timol. A composição química dos óleos essenciais de $O$. gratissimum relatada na literatura confirma a divisão estabelecida por Guenther ${ }^{10}$. Diversas plantas ricas em timol ${ }^{8,12,13,14,16,18}$, e ricas em eugenol $1^{6,7,9,11,22}$ tem sido relatadas na literatura. Mais recentemente, um terceiro tipo químico, rico em geraniol foi relatado 5 .

A familia Lamiaceae é também rica em muitos tipos de flavonóides, os quais tem sido frequentemente usados com propósitos quimiotaxonômicos ${ }^{20}$. Estes compostos são encontrados na superfície das folhas, ramos e inflorescências de plantas, e são armazenados em pelos glandulares especiais ${ }^{3}$.

O objetivo deste trabalho é determinar se estes metabólitos secundários podem ser usados como marcadores taxonômicos em $O$. gratissimum. Este trabalho pretente auxiliar na identificação taxonômica e diferenciação da variabilidade existente entre acessos de $O$. gratissimum.

${ }^{1}$ Este artigo representa uma parte do original publicado na íntegra na revista Biochemical Systematic and Ecology, 29/3: 287-304.

\section{Material e Métodos}

Material: Doze acessos de Ocimum gratissimum (Tabela 1) foram cultivados em Purdue University Research Station, West Lafayette, USA em experimento em blocos completamente ao acaso com quatro indivíduos em cada uma das três repetições. A planta inteira foi colhida de cada indivíduo em cada bloco em plena floração, colocadas em saco de papel e secas em estufa de ar circulante a $38^{\circ} \mathrm{C}$ por 15 dias para análise dos óleos essenciais. Excicatas de cada acessos foram coletadas e depositadas no herbário da Purdue University (POU). Duplicatas foram enviados aos herbários de Kew Gardens (K) e Delaware State University (DOL) para coleção permanente.

Extração e Análise dos Óleos Essenciais: Os óleos voláteis foram extraídos de amostras secas em balões de 21 com água destilada usando aparelho Clevenger, conforme descrito em Charles and Simon ${ }^{4}$. Os óleos essenciais foram analizados em Cromatografo a Gás modelo Varian, equipado com FID e integrador eletrônico 4270. Uma coluna capilar de sílica (30 m x 0,25 mm ID) com RSL-200 fase fixa (Altech, 5\% fenil, 95\% metilpolisiloxano) foi usada. Hélio foi usado como gás carreador e a temperatura do forno foi estabelecida à $80{ }^{\circ} \mathrm{C}$ por 2 min e programado para aumentar a $3{ }^{\circ} \mathrm{C} / \mathrm{min}$ à $160^{\circ} \mathrm{C}$. As temperaturas do injetor e detector foram de $180^{\circ} \mathrm{C}$ e $300^{\circ} \mathrm{C}$, respectivamente. A identificação dos constituites químicos dos óleos essenciais foi confirmada por cromatógrafo a gás acoplado a espectro de massas usando modelo $4000^{4}$.

Flavonóides: A extração, identificação e quantificação dos flavonóides foi realizada conforme descrito em Vieira ${ }^{21}$.

\section{Resultados e Discussão}

Houve uma interessante correlação entre os perfis dos constituintes dos flavonóides e dos óleos essenciais. Os acessos contendo altos teores de flavonóides (ot26 e ot65) apresentaram alto teor de timol, enquanto que os acessos contendo baixo teor de flavonóides (ot27, ot63, and ot85) tinham alto teor de eugenol (Tabela 2). O acesso ot84, que apresentou um teor intemediário de flavonóides, aprentou geraniol como seu principal constituinte do óleo essencial. É possível que os acessos ricos em eugenol contenham baixo teor de flavonóides porque eugenol, sendo um fenilpropanóide, pode competir com mais sucesso que os flavonóides pelo mesmo aminoácido precursor, a fenilalanina, da mesma via metabólica. Pode não haver o mesmo tipo de competição entre os flavonóides e timol e geraniol, ambos terpenos, oriundos da via do ácido mevalônico.

As observações morfológicas e de campo dos acessos de $O$. gratissimum nos permitiram identificar 6 acessos dentro desta espécie, baseado principalmente no indumento foliar e dos ramos, bem como formato da inflorescência. Diferenças no formato e tamanho das folhas também foram observadas (Tabela 3). A análise dos óleos essenciais de todos acessos nos 
Tabela 1. Lista de acessos de Ocimum gratissimum, origem, crescimento e teor de óleo essencial, cultivados na Purdue University, West Lafayette, IN, USA

\begin{tabular}{|c|c|c|c|c|c|c|}
\hline Acesso & Origem & Denominação do acesso & $\begin{array}{l}\text { Altura } \\
(\mathrm{cm})\end{array}$ & $\begin{array}{l}\text { Peso fresco } \\
\text { total }(\mathrm{g})\end{array}$ & $\begin{array}{l}\text { Peso seco } \\
\text { total }(\mathrm{g})\end{array}$ & $\begin{array}{l}\% \text { óleo essencial } \\
\text { (g peso seco) }\end{array}$ \\
\hline ot17 & USA & USDA $^{\mathrm{a}}$, PI211715 & $64 \pm 2,9$ & $536,5 \pm 90$ & $151,2 \pm 26,5$ & $0,72 \pm 0,11$ \\
\hline ot24 & Russia & BRA116 & $57,3 \pm 3,8$ & $422,8 \pm 96,1$ & $107,8 \pm 19,5$ & $0,31 \pm 0,04$ \\
\hline ot26 & Brazil & BRA043 & $59 \pm 3,6$ & $505 \pm 122$ & $125,9 \pm 29$ & $0,33 \pm 0,12$ \\
\hline ot27 & Brazil & BRA078 & $49 \pm 2,2$ & $441,5 \pm 51,5$ & $105,4 \pm 14$ & $0,49 \pm 0,09$ \\
\hline ot52 & USA & East Indian, Richters ${ }^{\mathrm{c}}$ & $63 \pm 3,2$ & $436 \pm 127,8$ & $113,5 \pm 31,5$ & $0,46 \pm 0,18$ \\
\hline ot63 & USA & Tree, Richters & $51,5 \pm 3$ & $280 \pm 72,8$ & $68,4 \pm 16,1$ & $0,45 \pm 0,08$ \\
\hline ot65 & USA & Green, Richters & $53,3 \pm 2,4$ & $326,8 \pm 21,2$ & $83,6 \pm 5,5$ & $0,44 \pm 0,13$ \\
\hline ot84 & USA & Purdue University (tipo geraniol) & $60,8 \pm 3$ & $618,3 \pm 77,5$ & $149 \pm 26$ & $1,66 \pm 0,62$ \\
\hline ot85 & USA & Purdue University (tipo eugenol) & $51,3 \pm 3,4$ & $500,5 \pm 280$ & $133,8 \pm 70,5$ & $0,50 \pm 0,22$ \\
\hline
\end{tabular}

${ }^{a}$ USDA, United States Plant Introduction Center, Ames, Iowa

b Embrapa, Empresa Brasileira de Pesquisa Agropecuária, Brasilia, Brasil.

c Richters Co., Goodwood, Ontario, Canada.

d Purdue University, West Lafayette, Indiana.

Tabela 2. Porcentagem relativa de flavonas do total de flavonóides presentes em cada extrato dos acessos de Ocimum gratissimum

\begin{tabular}{|c|c|c|c|c|c|c|c|}
\hline \multirow[b]{2}{*}{ Flavonóides $^{\mathrm{a}}$} & \multicolumn{7}{|c|}{ Raças químicas de Ocimum gratissimum } \\
\hline & $\begin{array}{l}\text { Eugenol } \\
\text { (ot27) }\end{array}$ & $\begin{array}{l}\text { Eugenol } \\
\text { (ot52) }\end{array}$ & $\begin{array}{l}\text { Eugenol } \\
\text { (ot63) }\end{array}$ & $\begin{array}{l}\text { Eugenol } \\
\text { (ot85) }\end{array}$ & $\begin{array}{l}\text { Geraniol } \\
\text { (ot84) }\end{array}$ & $\begin{array}{l}\text { Timol } \\
\text { (ot26) }\end{array}$ & $\begin{array}{l}\text { Timol } \\
\text { (ot65) }\end{array}$ \\
\hline xantomicrol & & & & & $8,75 \pm 6,9$ & $89,8 \pm 3,1$ & $86,8 \pm 0,5$ \\
\hline cirsimaritina & $100 \pm 0$ & $100 \pm 0$ & $100 \pm 0$ & $100 \pm 0$ & $66 \pm 4,4$ & $10,0 \pm 2,8$ & $11,8 \pm 1$ \\
\hline total de flavonóides $(\mathrm{mg} / \mathrm{g})$ & $0,03 \pm 0,01$ & $0,01 \pm 0,006$ & $0,01 \pm 0$ & $0,01 \pm 0$ & $0,43 \pm 0,1$ & $2,1 \pm 0,8$ & $1,43 \pm 0,3$ \\
\hline
\end{tabular}

${ }^{\text {a }}$ percentagem relativa de flavonóides; ${ }^{\mathrm{b}}$ valores relatados como média de tres repetições \pm Desvio Padrão

Tabela 3. Área foliar, pubescência das folhas e ramos e principais constituintes dos óleos voláteis relatados para diferentes tipos morfológicos de Ocimum gratissimum

\begin{tabular}{l|l|l|l|l}
\hline Acessos & Área foliar $\left(\mathrm{cm}^{2}\right)$ & Ramos & Folhas & Principais constituintes dos óleos voláteis \\
\hline ot17 & $15,2 \pm 3,3$ & piloso & piloso & eugenol / espatulenol \\
\hline ot24 & $53,1 \pm 24,9$ & glabro & glabro & timol / $\alpha$-copaeno \\
\hline ot27 & $70,9 \pm 11,1$ & piloso & piloso & eugenol / $\gamma$-muuroleno / espatulenol \\
\hline ot65 & $38,3 \pm 7,4$ & glabro & glabro & timol $/ \mathrm{p}$-cimeno \\
\hline ot84 & $35 \pm 9,9$ & piloso & piloso & geraniol \\
\hline ot85 & $11,4 \pm 3$ & piloso & piloso & eugenol / timol / espatulenol \\
\hline
\end{tabular}

permtiram identificar 3 tipos químicos de $O$. gratissimum (eugenol, timol e geraniol), os quais são possíveis de serem identificados pela análise organoléptica de cada acesso.

A similaridade entre os flavonóides e óleos essenciais reforça as observações feitas em campo. Metabólitos secundários, como os flavonóides e óleos essenciais, podem estar associados a alguns tratos morfológicos, e são úteis para compreender a variação existente dentro deste grupo.

Apesar deste estudo conter apenas uma pequena amostra de $O$. gratissimum, parece que os caracteres morfológicos são de difícil uso sozinhos como descritores, devido a sua contínua variação. A divisão da espécie em duas variedades com base apenas em morfologia, obscurece o alto grau de variação na produção de metabólicos secundários encontrada na var. gratissimum, em contrate do que foi encontrado na var. macrophyllum.

Enquanto os constituintes dos óleos essenciais e flavonóides exibem uma excelente discriminação entre os acessos de $O$. gratissimum, o uso de metabólitos secundários como marcadores intra-específicos per se necessitam considerar outros fatores, tais como fatores ambientais, desenvolvimento da planta, e método de extração utilizado, todos com grande impacto na composição dos óleos essenciais e flavonóides. Pino ${ }^{16}$ encontrou composição diferente dos óleos essenciais 
Tabela 4. Composição química dos acessos de Ocimum gratissimum, cultivados em condições de campo, Purdue Universisty, USA

\begin{tabular}{|c|c|c|c|c|c|c|c|c|c|c|}
\hline \multirow{2}{*}{$\begin{array}{l}\text { Constituintes do } \\
\text { óleo essencial }\end{array}$} & \multicolumn{10}{|c|}{ Acessos de $O$. gratissimum } \\
\hline & $\mathrm{TRb}$ & ot17 & ot 24 & ot 26 & ot 27 & ot52 & ot63 & ot65 & ot84 & ot85 \\
\hline & \multicolumn{10}{|c|}{ (porcentagem relativa do total de óleo essencial)c } \\
\hline p-cimeno & 5,9 & $0,3 \pm 0,2$ & $3,4 \pm 2,2$ & $2,8 \pm 1,7$ & & $0,3 \pm 0,3$ & $0,4 \pm 0,4$ & $9,6 \pm 4,5$ & $0.8+0.4$ & \\
\hline$\gamma$-terpineno & 6,74 & & $1,6 \pm 0,7$ & $2,3 \pm 0,7$ & $\mathrm{t}$ & $2 \pm 2,8$ & $0,1 \pm 0,2$ & $2,3 \pm 0,3$ & $\mathrm{t}$ & \\
\hline$\alpha$-terpineol & 7,22 & $2,3 \pm 0,2$ & $2,5 \pm 0,6$ & $2,4 \pm 1,3$ & $0,2 \pm 0,2$ & $3,2 \pm 1$ & $2,1 \pm 1$ & $4,3 \pm 0,4$ & $0.1+0.2$ & $0.4+0.4$ \\
\hline terpinoleno & 8,12 & $1,1 \pm 0,6$ & $2 \pm 1,4$ & $1 \pm 0,1$ & $0,8 \pm 0,4$ & $1,4 \pm 0,2$ & $1,4 \pm 0,7$ & $1,9 \pm 0,3$ & $0.6+0.1$ & $1.4+1.6$ \\
\hline terpinen-4-ol & 10,95 & $0,9 \pm 0,4$ & $1,4 \pm 0,4$ & $1,6 \pm 0,1$ & $\mathrm{t}$ & $1 \pm 0,3$ & $1,6 \pm 0,8$ & $2,6 \pm 0$ & $0.1+0$ & $\mathrm{t}$ \\
\hline metilchavicol & 11,72 & $0,5 \pm 0,4$ & $0,5 \pm 0,2$ & $0,6 \pm 0,2$ & $0,4 \pm 0,2$ & $0,8 \pm 0,4$ & $0,5 \pm 0,5$ & $1,7 \pm 0,5$ & $0.3+0.2$ & $0.6+0.3$ \\
\hline anisol & 14,08 & $0,9 \pm 0,6$ & $1,5 \pm 1,9$ & $7,3 \pm 7,6$ & $1,4 \pm 2,5$ & & $1,2 \pm 1,6$ & $2,2 \pm 1,9$ & & $1.9+1.4$ \\
\hline geraniol & 15,8 & & & & & & & & $85.4+2.1$ & \\
\hline timol & 16,39 & $5,2 \pm 3,5$ & $37,2 \pm 3,3$ & $38,5 \pm 5,1$ & $5,7 \pm 2,3$ & & $0,3 \pm 0.2$ & $44.3 \pm 0.8$ & $1.1+0.3$ & $12.7+7.5$ \\
\hline eugenol & 18,72 & $62,6 \pm 3,2$ & $3,5 \pm 1$ & $2,1 \pm 1,5$ & $45,1 \pm 8,6$ & $50,1 \pm 2,7$ & $44,1 \pm 10,5$ & $3,3 \pm 1,7$ & $1.1+0.8$ & $41.7+8.5$ \\
\hline$\alpha$-copaeno & 20,75 & $2,4 \pm 0,4$ & $14,3 \pm 2,3$ & $11,9 \pm 2,7$ & $1,5 \pm 0,4$ & $2,8 \pm 1,2$ & $1,8 \pm 0,3$ & $4,1 \pm 0,9$ & $1.3+0.1$ & $0.7+0.1$ \\
\hline$\beta$-cariofileno & 22,03 & $0,3 \pm 0,1$ & $1,9 \pm 0,2$ & $1,6 \pm 0,2$ & $1 \pm 0,4$ & $0,6 \pm 0,3$ & $0,3 \pm 0$ & $0,9 \pm 0,2$ & $0.8+0.1$ & $2.2+0.3$ \\
\hline$\gamma$-selineno & 23,66 & $1,4 \pm 0,6$ & $6,4 \pm 0,9$ & $5,1 \pm 1,1$ & $0,2 \pm 0,3$ & $1,3 \pm 1,2$ & $0,5 \pm 0$ & $6,8 \pm 1,4$ & $2.1+0.2$ & $0.3+0.4$ \\
\hline$\gamma$-muuroleno & 24,47 & $1 \pm 0,3$ & $6,4 \pm 0,7$ & $5 \pm 0,7$ & $20,2 \pm 2,2$ & $1,6 \pm 1,1$ & $1,1 \pm 0,5$ & $1,2 \pm 0,2$ & $0.4+0.1$ & $1.1+0.1$ \\
\hline espatulenol & 27,6 & $9,9 \pm 2,3$ & $4,3 \pm 0,9$ & $3,7 \pm 0,8$ & $14,3 \pm 3$ & $25,3 \pm 5,2$ & $23,4 \pm 6,8$ & $1,9 \pm 0,3$ & $0.7+0.3$ & $15.4+3.9$ \\
\hline
\end{tabular}

${ }^{a}$ Listados em ordem de tempo de retenção $(\min ) ;{ }^{\mathrm{b}} \mathrm{TR}=$ tempo de retenção;

${ }^{\mathrm{c}}$ valores relatados como média de três repetições \pm desvio padrão. $\mathrm{t}=$ traços $(<0.05 \%)$

quando comparando extração por arraste a vapor, extração por solvente ou $\mathrm{CO}_{2}$ supercrítico. A mesma amostra de $O$. gratissimum resultou em concentrações variadas de timol, apesar deste ser sempre o principal composto. Compostos minoritários, tais como $\mathrm{p}$-cimeno e $\beta$-cariofileno, foram detectados em arraste a vapor, mas somentes traços foram verificados quando a extração foi feita por solvente ou $\mathrm{CO}_{2}$ supercrítico. Diferentes técnicas de extração tem demonstrado significtivo impacto nas concentrações relativas dos constituintes dos óleos essenciais de espécies de Ocimum $^{5}$. Além de fatores ambientais e métodos de extração, a variação genética intra-específica pode promover a segregação de diversos copostos minoritários, tais como sesquiterpenos.

Apesar de $O$. gratissimum ser largamente distribuído em todo mundo, poucos esforços foram feitos para sua domesticação. Foi observado que acessos com alto teor de timol e eugenol, apresentaram diferenças quanto a composição e teor de sesquiterpenos (Tabela 3). Neste caso, pode-se levantar a hipótese de que foram selecionados organolepticamente para diferentes aromas e usos medicinais no caso do timol e eugenol. O alto teor de geraniol em ot84 é devido provavelmente a seleção para alto teor de óleo na Purdue University 5 . O uso de metabólitos secundários em taxonomia pode ser mais útil em espécies como O. gratissimum, que ainda não são domesticadas, ao invés de espécies que já foram submetidas a extensa seleção e melhoramento genético.

\section{Referências}

${ }^{1}$ Albuquerque U P, Andrade L H C. 1998a. El genero Ocimum L. (Lamiaceae) en el nordeste del Brasil. Anales Jard. Bot. Madrid 56(1), 43-64

${ }^{2}$ Albuquerque U P, Andrade L H C. 1998 b. Etnobotanica del genero Ocimum L. (Lamiaceae) en las comunidades afrobrasilenas. Anales Jard. Bot. Madrid 56(1), 107-118

${ }^{3}$ Bosabaldis A, Gabrieli C, Niopas I. 1998. Flavone aglycones in glandular hairs of Origanum $x$ intercedens. Phytochemistry 49, 1549-1553

${ }^{4}$ Charles D J, Simon J E, 1990. Comparison of extraction methods for the rapid determination of essential oil content and composition of Basil. Journal of American Society for Horticulture Science 115(3):458-462

${ }^{5}$ Charles D J, Simon J E, 1992. A new geraniol chemotype of Ocimum gratissimum L. Journal of Essential Oil Research 4(3), 231-234

${ }^{6}$ Chogo J B, Crank G. 1981. Chemical composition and biological activity of the Tanzanian plant Ocimum suave. Journal of Natural Products 44(3), 308-311

${ }^{7}$ Cortez D A G, Cortez LE R., Pessini GL, Doro D L, Nakamura C V. 1998. Analysis of essential oil of alfavaca Ocimum gratissimum L (Labiatae). Arquivos de Ciências da Saúde da UNIPAR, 2(2), 125-127

${ }^{8}$ Ekundayo O. 1986. Essential oils. VIII. Volatile constituents of the leaves of Ocimum viride. Planta Medica 3, 200-202

${ }^{9}$ Fun C E, Svendsen A B, Baerheim-Svendsen A. 1990. 
Composition of the essential oils of Ocimum basilicum var. canum and $O$. gratissimum L. grown on Ariba. Flavor and Fragrance 5(3), 173-177

${ }^{10}$ Guenther E. 1948. The Essential Oils. II. Van Nostrand Co., New York

${ }^{11}$ Jankovsky M, Taborsky J, Hubacek J, Hlava B. 1990. Volatile substances in basil (Ocimum gratissimum L.). Sbornik UVTIZ, Zahradnictivi 17(1), 59-68

${ }^{12}$ Jirovetz L, Buchbauer G, Ngassoum M B. 1998. Aroma compounds of leaf and flower essential oils of the spice plant Ocimum gratissimum L. from Cameroon. Ernahrung 22(9), 395397

${ }^{13}$ Ndounga M, Ouamba J M. 1997. Antibacterial and antifungal activities of essential oils of Ocimum gratissimum and $O$. basilicum from Congo. Fitoterapia 68(2), 190-191

${ }^{14}$ Ntezurubanza L, Scheffer J J C, Svendsen A B, BaerheimSvendsen A. 1987. Composition of the essential oil of Ocimum gratissimum grown in Rwanda. Planta Medica, 53(5), 421-423.

${ }^{15}$ Paton, A., 1992. A synopsis of Ocimum L. (Labiatae) in Africa. Kew Bulletin 47(3), 403-435

${ }^{16}$ Pino J A, Rosado A, Fuentes V. 1996. Composition of the essential oil from the leaves and flowers of Ocimum gratissimum L. grown in Cuba. J Essential Oil Res. 8(2), 139-141

${ }^{17}$ Pino J A, Garcia J, Martinez M A. 1998. A comparison between the oil, solvent extract and supercritical carbon dioxide extract of Ocimum gratissimum L. J. Essential Oil Res. 10, 575-577.

${ }^{18}$ Sanda K, Koba K, Nambo P, Gaset A. 1998. Chemical investigation of Ocimum species growing in Togo. Flavour and Fragance Journal 13(4), 226-232

${ }^{19}$ Sobti S N, Pushpangadan P, 1982. Studies in the genus Ocimum: Cytogenetics, breeding and production of new strains of economic importance. In: Cultivation and Utilization of Aromatic Plants. (Atal, C.K., Kapur, B.M., eds.), pp. 457-472. Regional Laboratory Council of Scientific and Industrial Research, Jammu-Tawi

${ }^{20}$ Tomás-Barberán F A, Gil M I. 1992. Chemistry and natural distribution of flavonoids in the Labiatae. In: Harley, R.M., Reynolds, T. (Eds.), Advances in Labiate Science, Royal Botanic Gardens, Kew, pp. 299-305

${ }^{21}$ Vieira R F, R Grayer, A Paton, J E Simon. 2001. Genetic diversity of Ocimum gratissimum L. based on volatile oil constituents, flavonoids and RAPD markers. Biochemical Systematics and Ecology 29 (3):287-304

${ }^{22}$ Zamureeenko V A, Klyuev N A, Dmitriev L B, Polyakova S G, Grandberg I I. 1986. Essential oil component composition in basils of the eugenol type. Izvestiya Timiryazevskoi Sel'skokhozyaistvennoi Akademii 2, 172-175 